\title{
Calibrant Free Sampling and Enrichment with Solid-Phase Microextraction: Computational Simulation and Experimental Verification
}

\author{
Md. Nazmul Alam¹, Luis Ricardez-Sandoval², Janusz Pawliszyn ${ }^{1 *}$,
}

${ }^{1 *}$ Department of Chemistry, ${ }^{2}$ Department of Chemical Engineering, University of Waterloo, Waterloo, N2L 3G1, Canada

Phone: 1- 519-888-4641. Fax: 1-519-746-0435. E-mail: janusz@uwaterloo.ca. 


\begin{abstract}
Despite the benefits of diffusion-based calibrant-free sampling based on solid-phase microextraction (SPME), this quantification approach is often underestimated due to an inadequate understanding of how extraction parameters influence the extracted amount and quantification of analytes. Currently, application of this approach for complex samples with binding matrix components is very limited. This study presents the development of a computational model that is used to identify the critical parameters for the diffusion-based sampling. Simulations are conducted under simultaneous variations in mass transfer and adsorptive surface binding constants, and the presence of a binding matrix component in the sample. The simulation results correlate well with previously reported experimental data, and improve the predictions when compared to previously introduced semi-empirical models. This work enhanced basic understanding of physical processes involved in analyte quantification with SPME, which is of benefit when performing experimental designs, particularly where traditional calibration methods are not suitable.
\end{abstract}

Keywords: Solid-phase microextraction; quantification, diffusive sampling; calibration; mathematical modelling and simulation. 


\section{INTRODUCTION}

The most common application of solid-phase microextraction (SPME) as a technique for sampling and sample preparation is based on the attainment of equilibrium between the extracted analyte in the fiber coating and the analyte dissolved in the sample. The equilibrium method of quantification has been recognized as a reliable and easy-to-use approach, utilizing SPME fibers such as poly(dimethylsiloxane) (PDMS), where extraction is known to occur via absorption. ${ }^{1}$ With the use of solid coatings for SPME such as Carboxen/PDMS (CAR/PDMS) and PDMS/divinylbenzene (PDMS/DVB), high extraction capacity or analytical sensitivity can be obtained. ${ }^{2}$ The main principle of analyte extraction by these solid coatings is assumed to follow the process of adsorption on the surface. ${ }^{3}$ For these solid coatings, however, equilibrium-based quantification is often not a practical approach, owing to the long equilibration periods that are required in some instances. In some cases, long extraction times may also cause sufficient accumulation of substances on the coating so as to initiate competition between analytes and/or interferences, leading to saturation and displacement effects. ${ }^{4-6}$

Diffusive sampling is a method in which analytes are collected by an extraction phase (or, extractant) at a rate controlled by molecular diffusion of analytes through the diffusion boundary layer formed around the extractant in the sample matrix. ${ }^{7,8}$ It has been shown previously that by exposing a SPME fiber directly to a sample matrix for a short period of time, diffusive sampling can be obtained. This diffusion-based rapid sampling with SPME was introduced with aims to provide a simple and rapid sample analysis method. ${ }^{7}$ To date, different formats of diffusive sampling devices have been applied towards analysis of air and water samples. ${ }^{9-12}$ The 
requirements for the diffusion-based quantification are: (i) the extractant must behave as a zero sink or perfect sorbent; (ii) the extraction is controlled by diffusion through a diffusion boundary layer formed around the extractant surface, which can be assured by a steady fluid flow condition that maintains an unchanged diffusion-layer thickness; (iii) mass uptake should be linear irrespective of changes to either sampling time or analyte concentration. In order to satisfy the above requirements, therefore, sampling time should be optimized in view of the respective analyte concentrations and sample velocities. ${ }^{13}$ The main advantage of the diffusion-based quantification method is that it does not require the use of calibration curves or internal standards; as such, analyte competition for the same adsorption site can be avoided. Thus far, diffusive SPME samplers have been designed using one of two different geometries; the open bed format, generally chosen for rapid sample analysis; and the fiber retracted into a needle device for time-weighted average (TWA) concentration measurements. ${ }^{14,15}$ For quantification with the open bed format, a semi-empirical equation based on the assumption of uniform thickness of the diffusion layer is used to calculate the diffusion layer thickness. ${ }^{7}$ However, this method introduces large errors in the calculations, since the diffusion layer thickness is not uniform around the fiber and dependent on the physical dimensions of the fiber coating, sample flow conditions, and the physicochemical properties of the analytes under study. ${ }^{16}$ To overcome this drawback, Chen et al. ${ }^{17}$ proposed a semi-empirical physical model to describe rapid SPME quantification, as shown in eq.1.

$$
n=k_{0} A_{S} C_{A} t
$$


where $n$ is the amount of analyte extracted, $t$ is the sampling time, $A_{s}$ is the surface area of the coating, $C_{A}$ is the bulk analyte concentration, and $k_{0}$ is the average mass-transfer coefficient. In this approach, the mass transfer coefficients were calculated from simple empirical correlations that do not consider the geometry of the fiber. Furthermore, the assumption of zero sink behavior in diffusive sampling may not be appropriate for all analyte and sample conditions. For instance, sorbent affinity (or the distribution constant, $K$ ) and capacity (maximum extracted amount), both factors which may influence the zero sink behavior of the coating, were not considered in previous models. Moreover, previous models cannot predict analyte displacement during SPME extractions, which might occur even at short sampling times for high analyte concentrations, as reported for PDMS/DVB coating use. ${ }^{18}$

The fiber-retracted-in needle sampler allowed for the sampler to be used in unsteady fluid velocity conditions. ${ }^{19}$ In this device, the extraction phase is retracted into the needle at a certain distance to create an artificial static domain in which the analytes are assumed to be transported only by diffusion. Indeed, accurate TWA concentrations have been reported for both air and water samples with the use of the retracted SPME device. ${ }^{20,21}$ However, in order for the retracted SPME device to be widely accepted, certain parameters need to be further evaluated, such as the effects of extraction phase geometry on extraction, the length and diameter of the diffusion path and needle housing, the presence of a binding matrix component, etc. For example, it is important to understand how the desorption of matrix-bound analytes during sampling contributes to mass transfer and quantification as compared to the initial free concentration of the analyte in the sample. Recently, Jiang et al. ${ }^{22}$ proposed a modified equation for analyte 
quantification in the kinetic regime with the presence of a binding matrix component. The modified equation requires calculation of diffusion layer thickness, which might not be accurately estimated since the thickness of the layer varies with the geometry of the extraction phase, and the layer is not uniform around the extraction phase.

Numerical modelling and computational simulations are often used as tools to explain the behavior of processes at low costs. In our previous study, a mathematical model was developed to provide insight on the mass transport and binding processes occurring in SPME. ${ }^{23}$ While most of the analysis in that study was undertaken under static conditions, the rapid sampling SPME method requires that the fiber be placed in a flowing stream of analyte (dynamic sampling), an aspect that was not considered in our previous work. As such, in order to correctly estimate extraction quantitatively, it is of the utmost importance that the effects of convective flow and analyte affinity on the magnitude of the transport-controlled extraction are predicted with accuracy.

The aim of this study is to present a computational model for diffusive sampling with an SPME coating placed in a flow through system, and retracted in a needle device, as depicted in Figure 1. The predictions obtained with the proposed model have been compared with the predictions reported by the empirical model proposed by Chen et al. ${ }^{17}$, and with experimental data reported in the literature. 
(a)

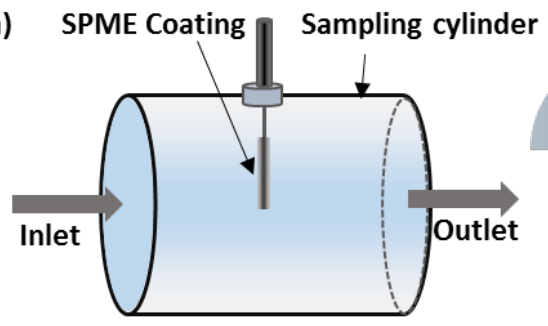

(c)

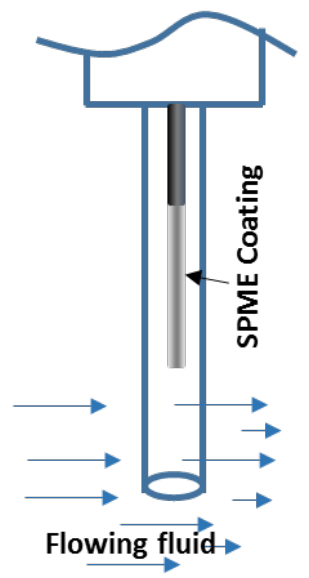

(b)

Cylinder wall/symmetry

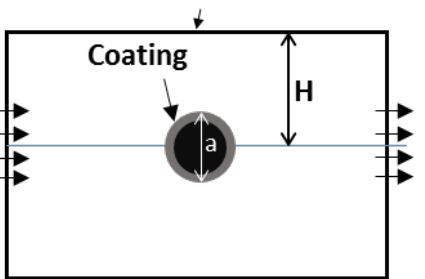

(d)
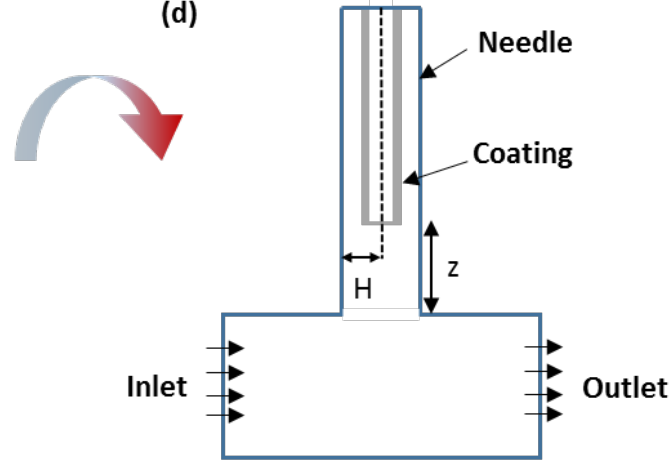

Figure 1. Schematic of experimental setup for diffusive-sampling in a flow-through system with SPME. (a) The sampling cylinder is used to mimic environmental sampling (e.g., river water) by rapid- SPME sampler. (b) Schematic of a 2D crosssection of the sampling cylinder and SPME fiber (not to scale). The fiber is located in the middle of the cylinder. Here, $H$ is the distance between the fiber center and the cylinder wall, and $a$ is the diameter of the fiber. (c) Schematic representation of a retracted SPME device. (d) The 2D geometry used in the numerical modeling of the fiber retracted in a needle device, where $H$ is the radius of the needle and $Z$ is the diffusion length.

\section{EXPERIMENTAL}

\section{The coupled mathematical model}

As depicted in Figure 1 (b) and (d), the model considered two dimensional segments of the two SPME systems. The velocity of the aqueous sample brings shear force to the coating-water interface, resulting in stronger convective forces and leading to a larger concentration gradient 
near the interface. Therefore, the flow in the sample domain is governed by the Navier-Stokes equations, while the flow field is treated as steady. ${ }^{24}$ Previous studies have shown that the Langmuir adsorption isotherm describes analyte extraction by solid coatings. ${ }^{3,25}$ The Langmuir isotherm model uses the active sites concept in the adsorption term to describe the effect of the adsorption rate as a function of the coverage of the coating. Therefore, this model has been used in the present study to develop the theoretical description of the adsorption process. Based on the Navier-Stokes equation, the solute transport equation, and the Langmuir equation, the coupled mathematical model can be established for the extraction process. Details of the model are shown in the supplementary information. A list of the model parameters used in this study is presented in Table S1.

\section{Numerical simulation}

COMSOL Multiphysics 5.1 was used to implement and solve the convection-diffusionreaction equations described above with a geometry representing a flow-through SPME sampling system containing a fiber vertically oriented to the flow (Figure 1b). A few assumptions were made to simplify the analysis for the fluid velocity and analyte concentration profiles in the system. First, the 3D flow-through geometry (Figure 1a and 1c) was reduced to the 2D cross section along the length of the channel, shown in Figure $1 \mathrm{~b}$ and $1 \mathrm{~d}$. This is an acceptable approximation when the fiber is located at the center of the cylinder, and the cylinder's walls are away from the fiber, i.e. there exists symmetry in the system. Since typical analytical samples have the bulk analyte concentrations in the sub-micromolar regime, the effect of mass transport on the fluid velocity is negligible; hence the simulations can be partitioned into two stages: (1) 
solution for fluid flow, and (2) solution of the coupled transient mass transport and surface reaction equations under a steady-state fluid flow assumption. In addition, the range of the flow velocities studied in this work were assumed to fall within the laminar flow regime. It is worthwhile to mention that the solid coatings used in SPME have micro or meso-porous structures. The porosity improves the extraction efficiency by increasing the number of active sites. We acknowledge that it is necessary that other key important effects be included in the present model, such as lateral interactions between the analyte and the extractant, migration effects, and many body and uncertainty effects resulting from surface microstructures. ${ }^{26,27}$ However, in this model, the micro-structure geometry was ignored due to the fact that our study was focused on the macro-scale kinetic feature of extraction, in which extraction is fully controlled by the diffusion layer. ${ }^{28}$ A refined mesh involving 92,000 finite elements was used in the present analysis. Increasing the number of elements does not improve the quality of the solution, but does increases the computational costs.

\section{RESULTS AND DISCUSSIONS}

\section{Diffusion based calibrations for rapid sampling}

After insertion of the SPME extractant into a sample, an initial rapid increase in mass uptake is followed by slow mass transfer kinetics to the extraction phase, until equilibrium is established between the extraction and sample phases. The analytical expression of time scale of extraction can be described using eq. $2^{25}$ 


$$
t_{e q} \approx t_{95 \%}=\frac{\delta_{s} K L}{D_{s} \Gamma_{\max }}
$$

where $\delta_{s}$ is the diffusion layer thickness, $L$ is the thickness of the extraction phase, $\Gamma_{\max }\left(\mathrm{mol} \mathrm{m}^{-2}\right)$ is the maximum surface concentration on the extraction phase and $D_{s}$ is the diffusion coefficient of the analyte in the sample matrix. The equilibration is only dependent on the equilibrium constant $(K)$ and diffusivity $\left(D_{s}\right)$ of the chemicals under study at a particular sample agitation (constant $\delta_{s}$ ) and a coating with known thickness. After the extraction reaches equilibrium between the extraction phase and the sample, the calibration process is rather simple; a discussion of this process can be found in detail elsewhere. ${ }^{2}$ In this work, however, calibration based on the initial linear uptake sorption regime can be predicted more accurately by employing the proposed mathematical model. Figure 2 shows the kinetic part of typical sorption time profiles obtained from the mechanistic mathematical model for constant concentrations of a few polycylic aromatic hydrocarbon (PAHs) in a continuous fluid flow condition, as depicted in Figure 1. As shown in Figure 2a, the extent of equilibration depends on the $K$ values obtained for the analytes; the higher the $K$ (which is equivalent to $\log P$ or hydrophobicity), the longer the equilibration time. Conversely, the extracted amounts for all four analytes were very similar up until twenty five minutes of extraction time had elapsed. Since the diffusion coefficients of the selected PAHs in water are very close to each other, the extracted amount is similar at the diffusion-controlled initial stages, when the coating is considered to be a zero sink. During this initial stage, the effect of equilibrium constants (or partition coefficients for liquid coatings, $K_{\text {es }}$ or $\log \mathrm{P}$ ) is negligible on the kinetics. This initial independence of $K$ on the extraction rate

\section{0 of 29}


introduces an interesting feature of SPME, by offering the possibility of calibration with only one calibrant. The minor variations in the diffusivities of the analytes result in uptake rate divergences that are within the expected experimental error (assuming 20\% error). For analytes with significantly different diffusion coefficients, calibration can only be obtained based on the diffusion coefficient. ${ }^{7}$ Figure $2 \mathrm{~b}$ shows an example of dependency of the extracted amount on the diffusion coefficient of the analytes. The results predicted by the model are in line with the experimental data reported by Chen et al. ${ }^{17}$ are also shown in the figure. 

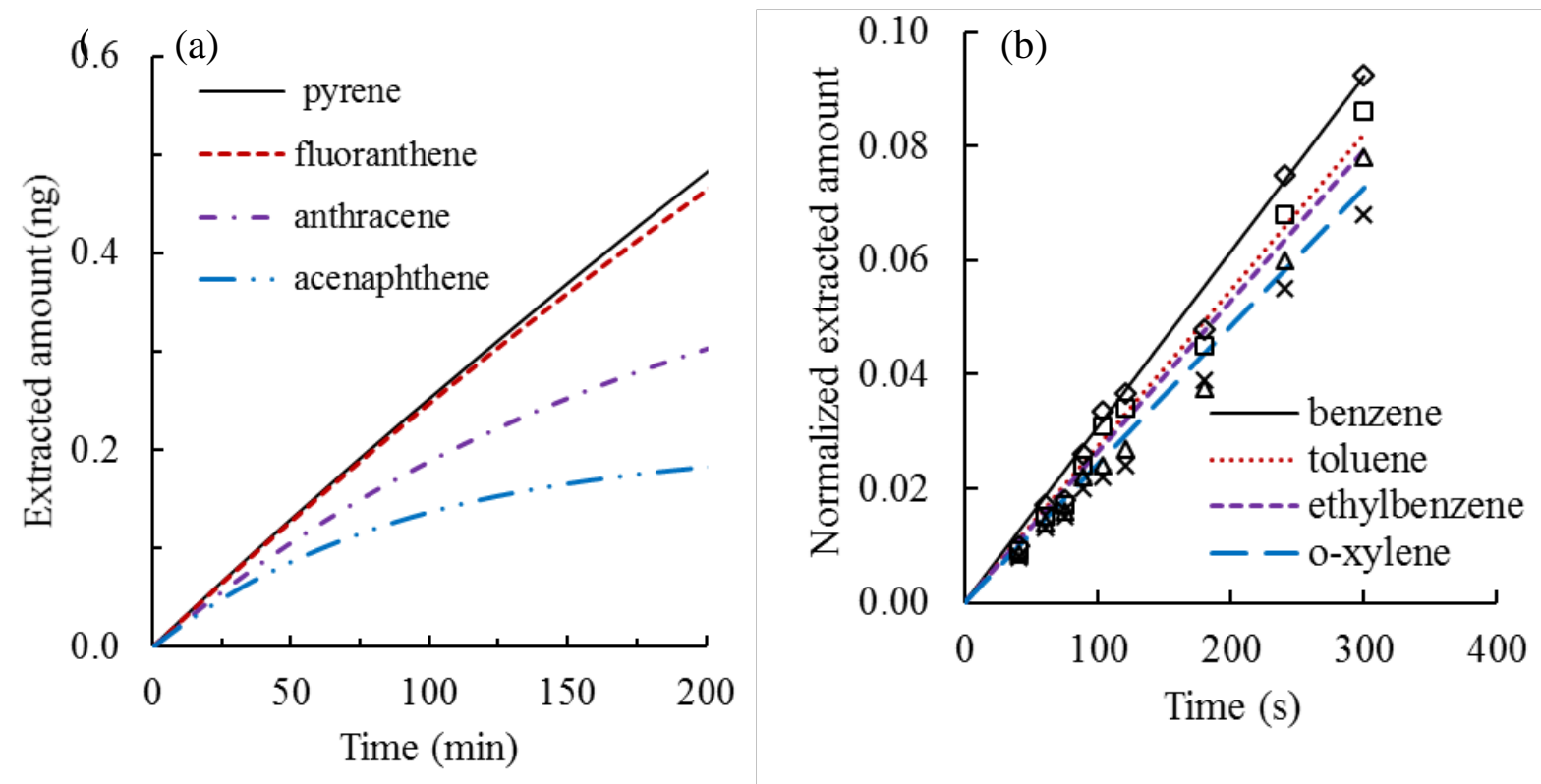

Figure 2. (a). Typical kinetic portion of the adsorption time profiles for PAHs, obtained from a simulation using the developed model. $D_{s}$ values are: $7.66 \times 10^{-6}$, $6.84 \times 10^{-6}, 6.59 \times 10^{-6}, 6.59 \times 10^{-6} \mathrm{~cm}^{2} \mathrm{~s}^{-1} ; K$ are $1 \times 10^{6} \mathrm{M}^{-1}, 2 \times 10^{6} \mathrm{M}^{-1}, 7 \times 10^{6} \mathrm{M}^{-1}$, $10 \times 10^{6} \mathrm{M}^{-1}$ for acenaphthene, anthracene, fluoranthene, and pyrene, respectively. $\Gamma_{\max }$ was set at $8 \times 10^{-5} \mathrm{~mol} \mathrm{~m}^{-2}$. (b). Comparison of simulated extraction time profiles with experimental profiles obtained from Chen et al. ${ }^{17}$ The lines are for simulated data, and symbols are for benzene: $\diamond$; toluene: $\square$; ethylbenzene: $\Delta ; \circ-x y l e n e: \times$. Normalized extracted amount was calculated by dividing the extracted amount with the amount extracted at equilibrium. Assumptions: concentrations of all analytes were $20.8 \mathrm{ng} / \mathrm{mL}$, fluid linear velocity of $0.2 \mathrm{~cm} / \mathrm{s}$ using a $75-\mu \mathrm{m}$ CAR/PDMS fiber. $\Gamma_{\max }$ and $K$ values are assumed as $1 \times 10^{-5}$ $\mathrm{mol} / \mathrm{m}^{2}$ and $\times 10^{8} \mathrm{M}^{-1}$, respectively.

\section{Effect of fluid flow on rapid diffusive sampling with SPME}

One of the requirements for rapid diffusive sampling is that the sample flow velocity must not be modified during the sampling time. The proposed model was employed to study the effect of flow velocity on the diffusion layer, and on the extraction kinetics of rapid sampling.

\section{2 of 29}


Numerical simulation results can provide insight into the dependency of the diffusion layer geometry on the sample flow velocity. Figure 3a shows the concentration profiles in the sample (2D) solution domain corresponding to the center of the fiber at various fluid flow velocities. The normally symmetrical diffusion layer obtained from static (diffusion only) conditions (Figure 3a I) is distorted when the convective flow is considered (Figure 3a II). The flow compresses the diffusion layer about the upstream edge (entrance to the fiber) of the fiber, whereas expansion of the diffusion layer is observed downstream of the fiber, i.e. at the outlet. The average thickness of the diffusion layer is dependent on the solution's inlet flow rate, being considerably thinner at faster flow rates in relation to the slower flow rates (Figure 3a III).

To quantify the role of transport in isolation to the adsorption of analytes onto the coating surface, assumptions of perfect and rapid adsorption kinetics were considered by setting very high sorption rate constants. Furthermore, the concentration of surface active sites $\left(\Gamma_{\max }\right)$ was considered to be very high $(0.1 \mathrm{~mol} \mathrm{~m})^{2}$. Therefore, analyte molecules are transported by a combination of convection and diffusion towards the coating surface where analytes are absorbed immediately and the surface never saturates. The benefit in considering this simplified case study is that analyte transport can be quantified in isolation from binding or saturation considerations. ${ }^{29}$

In a typical SPME sampling, the ratio of the sample (cylinder or a vessel) diameter to the fiber diameter is high; hence, the downstream convection prevents rapid growth of the diffusion layer to the cylinder wall. Consequently, the analyte species that interact with the fiber are confined to a thin layer near the fiber coating. For such flow conditions, the flow is approximated 
by a linear shear flow on the fiber. ${ }^{30}$ The mass transfer can then be characterized by a single dimensionless parameter, referred to as the shear Peclet number, $P e_{s}$ which is defined as follows:

$$
P e_{s}=\frac{u a^{2}}{D_{S} H}
$$

where $\boldsymbol{u}$ is the inlet velocity, $a$ is the fiber diameter, $H$ is the cylinder radius, and $D_{S}$ is the diffusion coefficient in sample. The thickness of the boundary layer, which is denoted as $\delta_{s}$, can be calculated as follows:

$$
\delta_{s}=\frac{a}{P e_{s}^{1 / 3}}
$$

As shown in eq. (3), $P e_{s}$ indicates whether the depletion zone is thick or thin relative to the coating diameter. The rate of mass transport through the depletion zone to the coating surface can be generalized as a dimensionless flux function, $F$ (also known as the Sherwood number), which is defined as follows:

$$
F=\frac{J_{D}}{D_{S} C_{A}}
$$

where $J_{D}$ is the total diffusive analyte flux to the coating surface, quantified through integration of the flux density at the coating surface. The dimensionless flux, $F$, was calculated by obtaining the analyte flux from the numerical simulation study. To evaluate the effect of fluid velocity and diffusion coefficient on the extraction of analytes from water, sample velocities and and diffusion coefficients ranging from $5 \times 10^{-4}$ to $4 \times 10^{-1} \mathrm{~m} \mathrm{~s}^{-1}$ and $2 \times 10^{-9}-2.5 \times 10^{-12} \mathrm{~m}^{2} \mathrm{~s}^{-1}$, respectively, were considered at 300 seconds of extraction, as shown in Figure 3b. The diffusion

\section{4 of 29}


range considered in the model simulation was very high to include analyte molecules of all sizes (small to large molecules, for example proteins) and gaseous or liquid samples. 
(a)

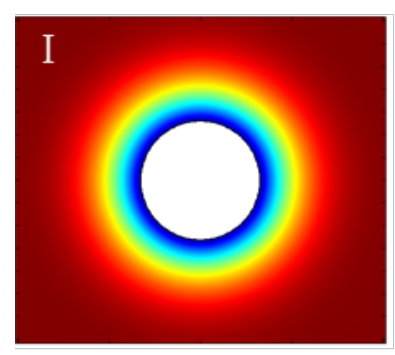

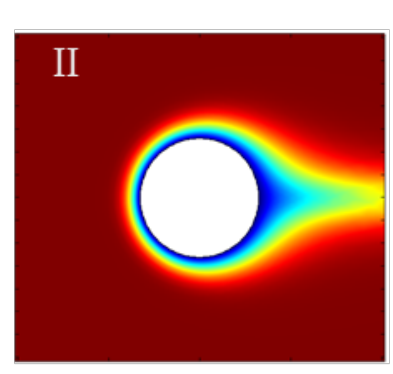
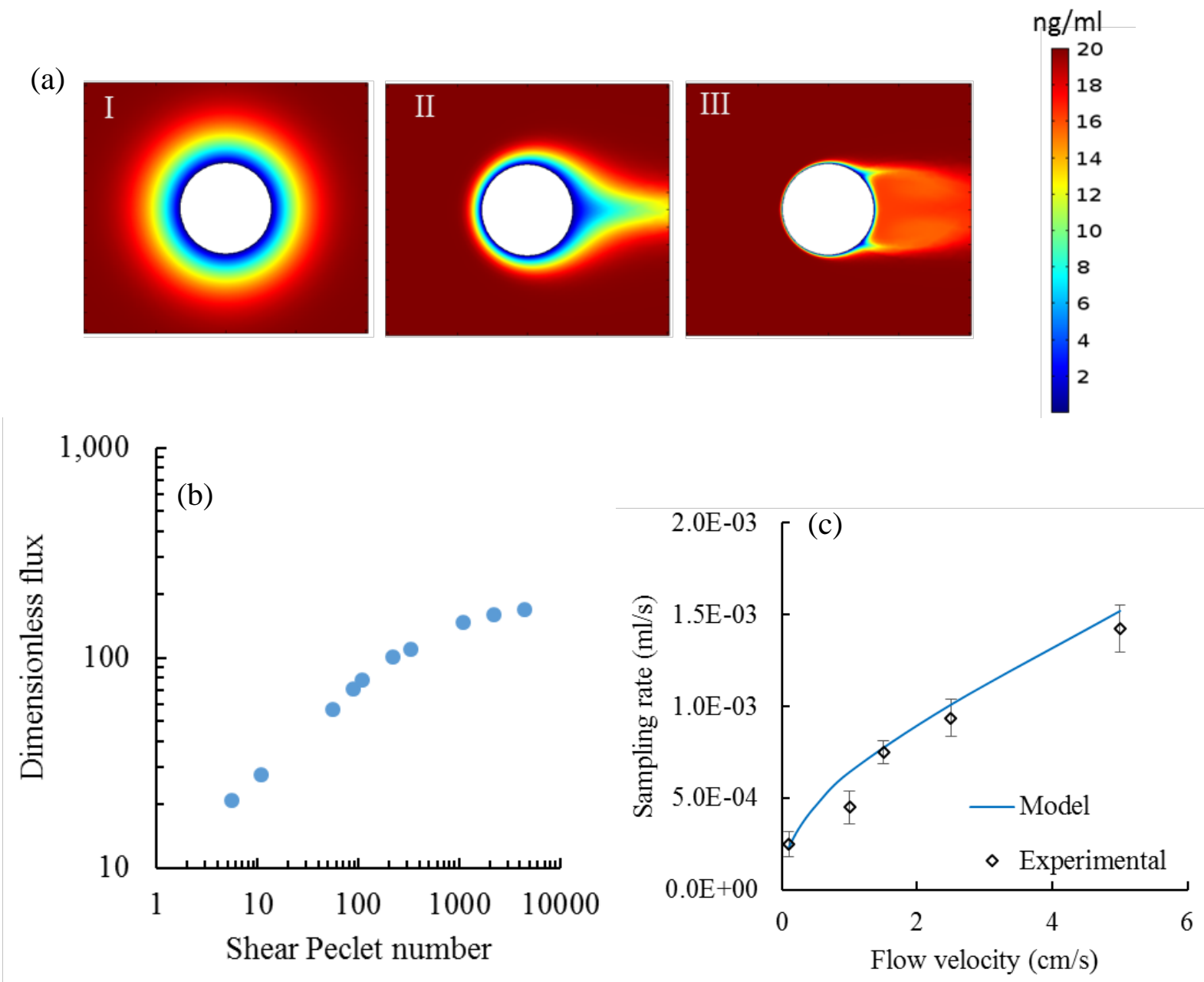

Figure 3. (a) Surface concentration showing the effect of fluid flow on the concentration boundary layer around the fiber, at 5 seconds of extraction. (I) A diffusion-only trial, with flow velocity of $0 \mathrm{~cm} \mathrm{~s}^{-1}$, (II) with flow velocity of $0.2 \mathrm{~cm}$ $\mathrm{s}^{-1}$, (III) with flow velocity of $10 \mathrm{~cm} \mathrm{~s}^{-1}$. Initial analyte concentration was considered $20 \mathrm{ng} \mathrm{ml}^{-1}$ for all simulations. (b)Effect of mass transport in terms of Peclet number $\left(P e_{s}\right)$ on the dimensionless flux $(F) . F$ was calculated from the total diffusive flux obtained from the model (see eq. 5). Here, the inlet velocity $(\boldsymbol{u})$ ranges from $5 \times 10^{-4}$ to $4 \times 10^{-1} \mathrm{~m} \mathrm{~s}^{-1}$, diffusivity $\left(D_{S}\right)=2 \times 10^{-9}-2.5 \times 10^{-9} \mathrm{~m}^{2} \mathrm{~s}^{-1}, H=1.5 \mathrm{~cm}, C_{A}=20.8 \mathrm{ng}$ $\mathrm{mL}^{-1}$ and $K=1 \times 10^{12} \mathrm{M}^{-1}$. (b) Comparison of experimental results with simulated data for the effect of flow velocity on sampling rate. ${ }^{17}$ Here, the sampling rate was obtained by the slope of the extracted amount (ng) devided by the sample initial concentration (ng ml$l^{-1}$ ) and the time (s). 
The dimensionless flux increases as the Peclet number increases; $P e_{s}$ is also correlated with eq. 4 , where increasing $P e_{s}$ reduces the boundary layer thickness, which in turn enhances the flux. At high Peclet numbers, the depletion zone becomes thinner than the coating thickness. In the lower range of $P e_{s}$, a significant effect on the flux change was observed, which is reflected by the reduction of the thickness of the boundary layer. Therefore, at a very high fluid velocity, the extraction can be transformed from a transport-limited regime to a reaction (adsorption) rate limited regime. In the higher range of $P e_{s}$, a lesser effect is observed, where a nonlinear relationship between the flux and $P e_{s}$ is noted. In Figure 3c, a reasonably accurate correlation of the experimental data to the model simulation results is observed. ${ }^{17}$

Variations in analyte uptake rate along the fiber orientation were studied with the model, and can be seen in Figure 4. The development of a low pressure gradient at the back of the fiber caused the uptake rate to be lower than that of the front of the fiber. This effect of uptake rate variation proportionally increases with the flow rate. Although the flow through system with inflow and outflow was considered in the model, the flow can also be assumed normal to the fiber in a sample vial with a magnetic stirrer. We acknowledge that the flow regime might be turbulent for high stirring rate in a sample vessel with a fixed sample volume or in a stream of very high fluid velocity. As our only goal in this section of the manuscript was to explore the mass transport around the fiber under a laminar flow assumption, the model developed here is not appropriate to study turbulent flows. Nevertheless, the simulation results indicate that the formation of eddies at the back of the fiber enhances the analyte flux compared to the front of the fiber. Therefore, for experiments at flow velocities higher than about $10 \mathrm{~cm} \mathrm{~s}^{-1}$, rotation of the 
fiber will aid in fully exploiting the capacity of the fiber coating, thus producing consistent results.In practice fiber rotation can be performed by either manually or with an electric motor.
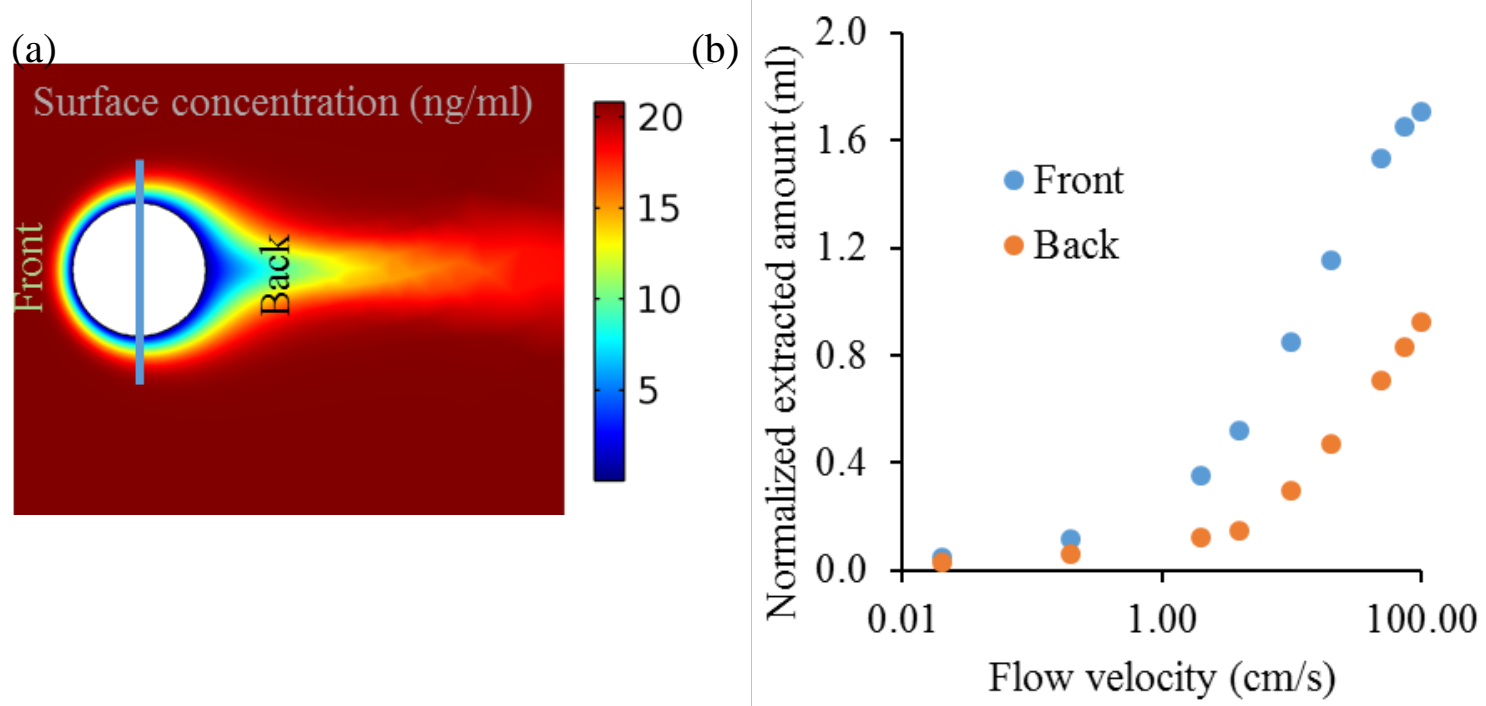

Figure 4. (a) Surface analyte concentration at the front and back of the fiber at the initial linear extraction regime. (b). Correlation between sample flow velocity and extracted amount, normalized by the initial concentration $\left(\mathrm{ng} \mathrm{ml}^{-1}\right)$ of the analyte. Benzene was chosen as a model analyte for the simulation, with a flow velocity of $2 \mathrm{~cm} \mathrm{~s}^{-1}$, with all other parameters shown in Figure 2.

\section{Effect of fluid flow on retracted SPME devices}

SPME in the retracted format is designed in such a way that the uptake rate is unaffected by the fluid velocity at the face of the needle. As shown in the supplementary material section (see Figure S2 a), sampling times are sensitive to variations in the diffusion coefficient of the analyte, where increasing the diffusion coefficient increases the sampling rate. The time necessary to reach a steady state in the diffusion path, which is referred to as the response time, depends on the path length and geometry of the fiber, as well as the diffusion coefficient of the analyte. 
Response time can be up to an hour based on the particular application being undertaken; however, since the sampling time is usually in the order of weeks and months, the length of the response time does not affect the performance of the device since the transients have already decayed (see Figure S2 b).

Therefore, the parameters that affect the adsorption process, including sample flow velocity, extractant maximum adsorption capacity, and analyte concentration, were investigated with the model described in this work. The model was employed to predict the sampling rate in consideration of variations in needle geometry. As shown in Figure 5, the sampling rate remains unaffected with changes in sample velocity for a small radius of the needle $(H)$ and long diffusion length $(Z)$. As the extraction phase is brought closer to the needle face and the needle diameter increases, the rate is gradually influenced by the flow velocity. Therefore, the model can aid in the selection of sampler geometry, aiding in the attainment of accurate quantification based on retracted SPME devices. 

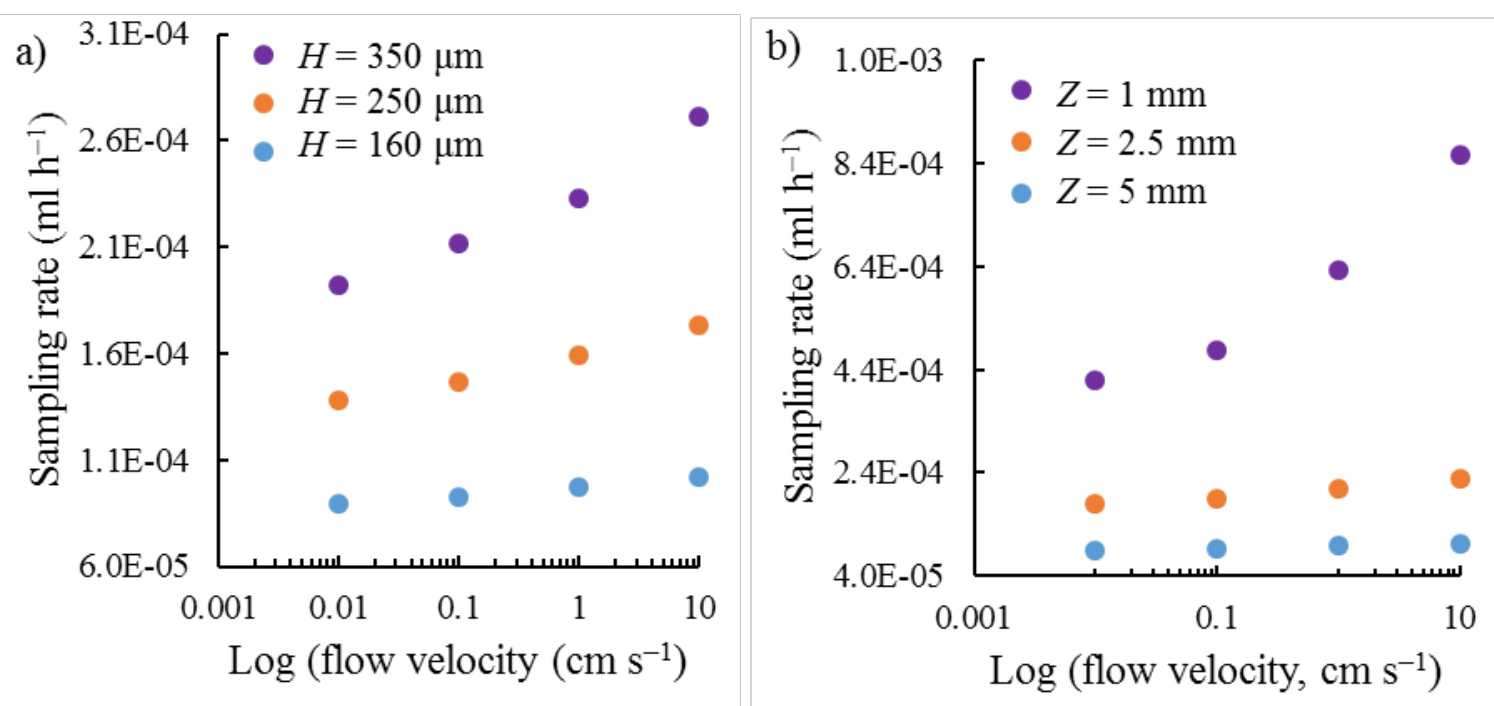

Figure 5. Effect of face flow velocity on the sampling rate for different sizes of the needle device. The needle radius $(H)$ was from 160 to $350 \mu \mathrm{m}$ and diffusion path $(Z)$ was varied from $1-5 \mathrm{~mm}$ (see Figure 1 for $H$ and $Z$ ). SPME fiber radius was $130 \mu \mathrm{m}$ and benzene was considered as a model analyte, with parameters described in Figure 2. Here, the sampling rate was obtained by the slope of the extracted amount (ng) devided by the sample initial concentration (ng ml${ }^{-1}$ ) and the time (h). 


\section{Effect of binding matrix components on diffusive sampling}

Analyte molecules present in a real sample might be bound with some other natural species, often called a binding matrix component. ${ }^{28}$ The most common binding principle is the reversible binding between the analyte $(A)$ and matrix component $(B)$ dominated by the association constant $\left(K_{a}\right){ }^{23}$ Binding of the analyte with the matrix results in a decrease in its free concentration in the sample. The reduced free concentration will provide a lower mass flux to the extraction phase. It has been assumed in the model that matrix components do not physically adsorb to the coating surface. As shown in Figure 6, the sampling rate with the rapid diffusive sampler decreases with an increase in the binding extent. The decrease in sampling rate is even more pronounced for higher concentrations of the matrix component. Therefore, ignoring the analyte binding phenomena will provide poor quantification with a diffusive sampler. 


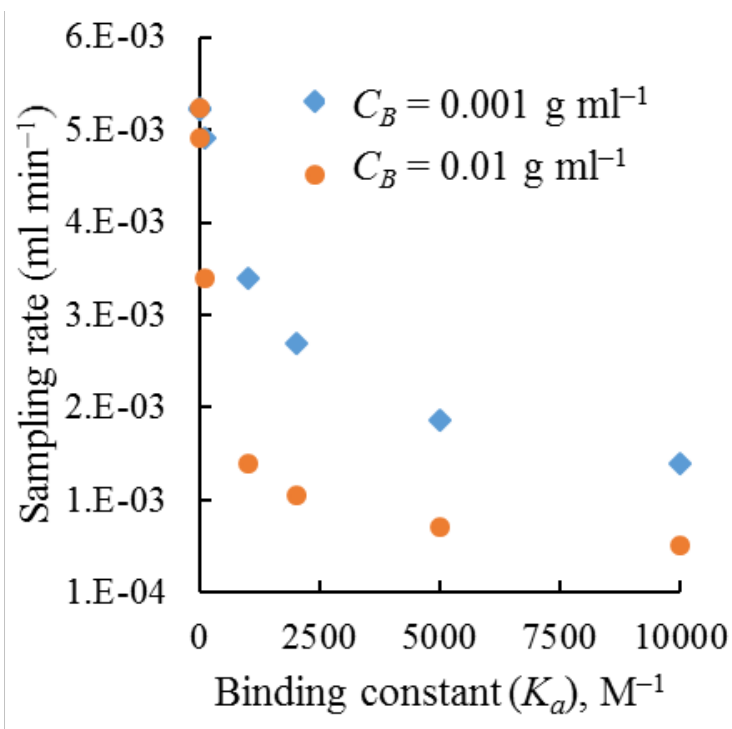

Figure 6. Effect of a binding matrix component on the sampling rate at two different matrix (B) concentrations. Total analyte concentration was kept at $20 \mathrm{ngml}^{-1}$ for all model experiments. $K_{a}$ values were altered by changing the forward rate constants, while the reverse rate constant was kept at $0.2 \mathrm{~s}^{-1}$.

Studies of the influence of matrix components on the analyte uptake kinetics is an active area of research. ${ }^{22,31-33}$ However, there is still a lack of understanding on the dynamics of such a multi-physics system for diffusive sampling with SPME. In this work, we have studied the effect of simultaneous variations of uptake kinetics and binding kinetics, as shown in Figure 7. The analyte uptake rate constant by the coating was set at a value of $10^{9} \mathrm{M}^{-1} \mathrm{~s}^{-1}$ (assuming very high analyte affinity for the coating ${ }^{34}$ ) and a range of dissociation rate constants were considered, with the highest value of $10^{-11} \mathrm{~s}^{-1}(\log K=20)$ being representative of a coating with very high affinity, and the lowest value, of $10^{5} \mathrm{~s}^{-1}(\log K=2)$, representing a weak extracting phase. The effect of six hypothetical ligands (Table 1) on analyte $(A)$ uptake by SPME was modeled with varying the association constant $\left(K_{a}\right)$ of the analyte-binding matrix component $(A-B)$. The 
association constant of the analyte with the matrix component, $K_{a}$, was varied from $10^{6}$ to $10^{14}$ $\mathrm{M}^{-1}$ to represent a range of dissociation rates, encompassing very labile to virtually inert conditions. In fact, changing the $K_{a}$ values has an effect on the equilibrium concentrations of the different species. As the focus of this work was the effect of the binding matrix component, its concentration was kept constant while the analyte and analyte-matrix component complex concentrations were appropriately adjusted to maintain equilibrium. The total concentration of analyte in the system was kept constant.

Table 1. Model parameters for studying the effect of binding matrix components on the diffusive sampling.

\begin{tabular}{|c|c|c|c|c|c|c|c|c|c|}
\hline & \multicolumn{2}{|c|}{$\begin{array}{l}\text { Diffusion coefficients } \\
\left(\mathrm{cm}^{2} \mathrm{~s}^{-1}\right)\end{array}$} & \multicolumn{2}{|c|}{ rate constants } & \multirow{2}{*}{$\begin{array}{l}\text { Associate } \\
\text { constant } \\
K_{a}\left(\mathrm{M}^{-1}\right) \\
\end{array}$} & \multicolumn{4}{|c|}{ reactant concentrations $\left(\mathrm{mol} \mathrm{L}^{-\mathrm{L}}\right.$ ) } \\
\hline & coating & sample & $k_{f}\left(\mathrm{M}^{-1} \mathrm{~s}^{-1}\right)$ & $k_{r}\left(\mathrm{~s}^{-1}\right)$ & & $C_{A}$ & $C_{B}$ & $C_{A B}$ & $C_{A}^{\text {total }}$ \\
\hline$B-1$ & $1.1 \times 10^{-6}$ & $1.1 \times 10^{-5}$ & $1.0 \times 10^{9}$ & $1.0 \times 10^{3}$ & $1.0 \times 10^{6}$ & $1.01 \times 10^{-7}$ & $1.0 \times 10^{-7}$ & $1.01 \times 10^{-11}$ & $1.01 \times 10^{-7}$ \\
\hline$B-2$ & $1.1 \times 10^{-6}$ & $1.1 \times 10^{-5}$ & $1.0 \times 10^{9}$ & $1.0 \times 10^{1}$ & $1.0 \times 10^{8}$ & $1.00 \times 10^{-7}$ & $1.0 \times 10^{-7}$ & $1.00 \times 10^{-9}$ & $1.01 \times 10^{-7}$ \\
\hline$B-3$ & $1.1 \times 10^{-6}$ & $1.1 \times 10^{-5}$ & $1.0 \times 10^{9}$ & $1.0 \times 10^{-2}$ & $1.0 \times 10^{11}$ & $9.18 \times 10^{-9}$ & $1.0 \times 10^{-7}$ & $9.18 \times 10^{-8}$ & $1.01 \times 10^{-7}$ \\
\hline$B-4$ & $1.1 \times 10^{-6}$ & $1.1 \times 10^{-5}$ & $1.0 \times 10^{9}$ & $1.0 \times 10^{-3}$ & $1.0 \times 10^{12}$ & $1.00 \times 10^{-9}$ & $1.0 \times 10^{-7}$ & $1.00 \times 10^{-7}$ & $1.01 \times 10^{-7}$ \\
\hline$B-5$ & $1.1 \times 10^{-6}$ & $1.1 \times 10^{-5}$ & $1.0 \times 10^{9}$ & $1.0 \times 10^{-4}$ & $1.0 \times 10^{13}$ & $1.01 \times 10^{-10}$ & $1.0 \times 10^{-7}$ & $1.01 \times 10^{-7}$ & $1.01 \times 10^{-7}$ \\
\hline$B-6$ & $1.1 \times 10^{-6}$ & $1.1 \times 10^{-5}$ & $1.0 \times 10^{9}$ & $1.0 \times 10^{-5}$ & $1.0 \times 10^{14}$ & $1.01 \times 10^{-11}$ & $1.0 \times 10^{-7}$ & $1.01 \times 10^{-7}$ & $1.01 \times 10^{-7}$ \\
\hline
\end{tabular}

Decreasing $\log K$ from 20 to 3 (Figure7a) had no effect on the analyte uptake by the binding components for the rapid diffusive SPME sampler. However, changing the dissociation rate of the complex in solution by changing its association constant (matrix components 1-6, Table 1) had a large effect on the uptake (Figure 7a). The accumulated mass of analyte was more affected by $K$ for systems with stronger binding ligands in the solution. As $\log K$ was decreased to less than 3, the amount of analyte-binding matrix complex formed decreased, especially for the systems with the most stable binding components ( $B-5$ and $B-6$ in Table 1$)$, due to increased competition for the free analyte by the binding matrix component.

\section{3 of 29}


The influences of the input parameters (Table 1) on the amount of analyte extracted with a fiber in a needle device are shown in Figure $7 \mathrm{~b}$. Similar to the rapid sampling device, the uptake rate remained unchanged for $\log K>3$, being only negatively dependent on the strength of the association constant, $K_{a}$. Note that an increased uptake rate was obtained for low $\log K$ values $(\log K<3)$ and with labile analyte-matrix complexes. This increased rate for labile complexes in solution is owed to the influence of the extra flux obtained from the desorbed analytes in the diffusive path of the sampler. This study reveals an important feature of SPME related to its ability to extract a wide range of analytes with different $\log P$ with a single experiment. This feature is also referred to as balanced coverage. ${ }^{6}$ Since analytes with higher $\log P$ values tend to have both higher $K$ and $K_{a}$ values, the reduced uptake rate forbids them from saturating the fiber coating. 

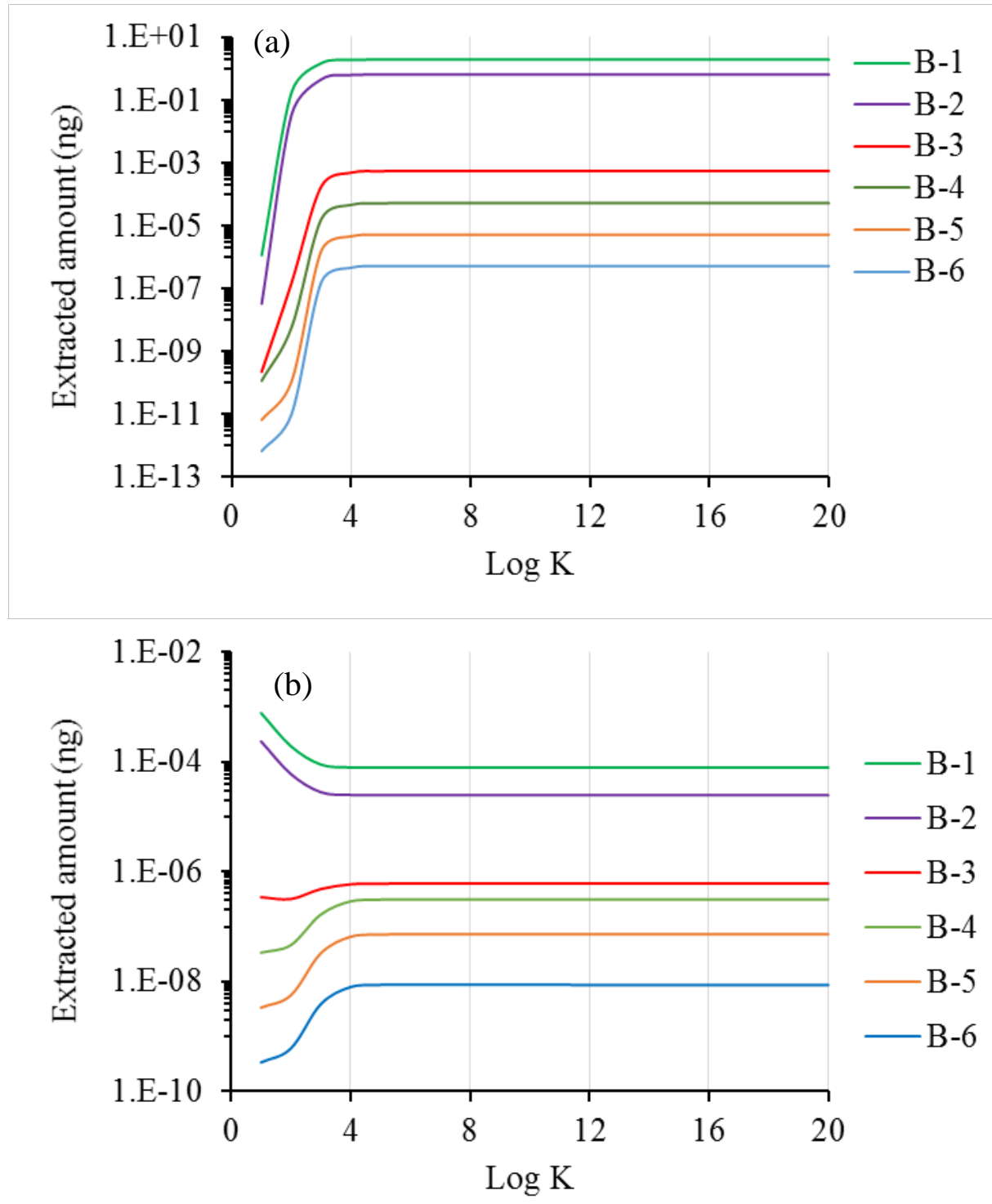

Figure 7. Effect of variation in the association constant of the matrix binding component on SPME uptake in the presence of binding components of varying lability (Table 1). Two different diffusive samplers are studied; rapid diffusive sampler (a) and retracted SPME sampler (b). For this simulation the flow velocity was set at $2 \mathrm{~cm} \mathrm{~s}^{-1}$.

\section{MODEL VALIDATION}


The mathematical model proposed in this work was compared with the model previously reported by Chen et al. (see eq. 1) and with experimental data for rapid water sampling of benzene, reported in same study. ${ }^{17}$ As illustrated in Figure 8, and from the error analysis (Table S2), the computational model proposed in this work predicted more accurately the mass uptake than the previous model available in the literature. ${ }^{17}$ In Chen's model, an accurate solution was not available due to the difficulty of estimating $k_{0}$; hence, an empirical correlation was used. On the other hand, the present computational model can capture the complex multi-phase extraction process, as most of the data is imbued with lower root mean square values (Table S2). In addition, the present model describes an idealized physical mass-transfer process using a mechanistic model, in which model parameters have a physical meaning; hence, the benefits of the present mathematical model.

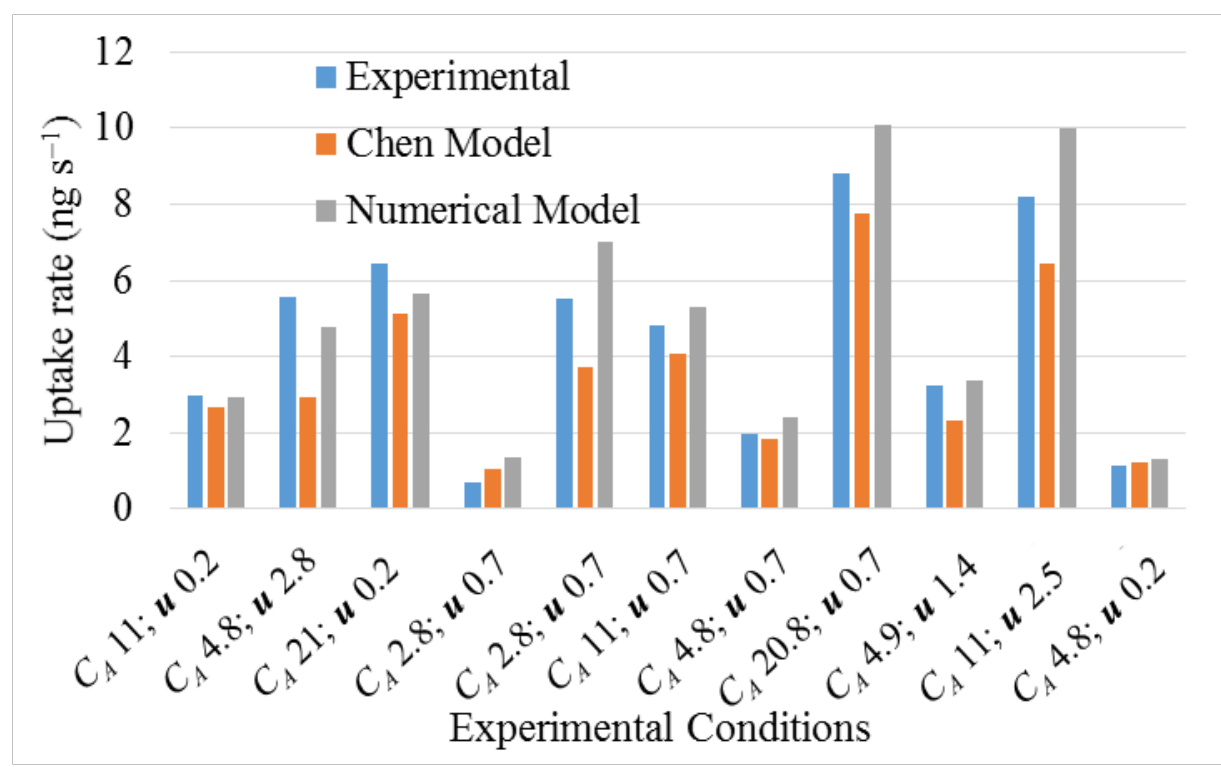

Figure 8. Model validation simulation data obtained from the developed computational model and comparisons to both the Chen model and experimental data presented in the literature ${ }^{17} . C_{A}$ and $\boldsymbol{u}$ stand for the concentration $\left(\mathrm{ng} \mathrm{ml}^{-1}\right)$ of benzene 
as a model analyte and flow velocity $\left(\mathrm{cm} \mathrm{s}^{-1}\right)$, respectively, used in the studies.

\section{CONCLUSIONS}

Experimental parameters that influence quantification of diffusive sampling devices based on SPME have been studied by developing a computational model that captures the involved processes. The model was validated using experimental data reported in the literature. The amount of extracted mass predicted by the present model compares very well with experimental mass uptakes. The model demonstrated excellent prediction capabilities in terms of the effect of mass transfer caused by the variation of fluid flow on the extraction rate of the SPME sampling device. The diffusive sampling approach with a given sampler geometry was shown to be dependent only on the diffusion of the analyte. Therefore, with the aid of the model, estimations of sampling time and sampling rate are possible within a short time and at low costs, i.e. without the need to perform wet experiments. In addition, adequate selection of flow velocity and required extraction capacity can be performed for a wide range of analytes with varied equilibrium constant values $(K)$. Simulation results showed that in the presence of a binding matrix, the sampling rate decreases compared to a sample with no binding matrix. This study revealed that rotation of the fiber should be implemented during sampling in order to utilize both sides of the fiber. It has been shown that extraction by an SPME device can cover a wide range of analytes, even if there is a binding matrix with varied binding affinity. Overall, the simulation results provide a detailed understanding of extraction parameters for SPME extraction using 
porous particle based solid coatings. In the future, in-silico optimization of experimental conditions for both liquid and solid coatings will be performed with the aid of the present computational model.

\section{ASSOCIATED CONTENT}

\section{Supporting Information}

Detail mathematical model for analyte transport and adsorption, concentration profiles (S1) and extraction time profiles (S2) in the retracted device, model parameters used (Table S1) and quantitative evaluation of the numerical model (Table S2).

\section{Acknowledgements}

The authors are thankful to the Natural Sciences and Engineering Research Council of Canada -Industrial Research Chairs Grants (NSERC-IRC) and the Ontario Research Funding (ORF) for financial support.

References

(1) Poerschmann, J.; Górecki, T.; Kopinke, F.-D. Sorption of Very Hydrophobic Organic Compounds onto Poly(dimethylsiloxane) and Dissolved Humic Organic Matter. 1. Adsorption or Partitioning of VHOC on PDMS-Coated Solid-Phase Microextraction Fibers - a Never-Ending Story?. Environ. Sci. Technol. 2000, 34, 3824.

(2) Ouyang, G.; Pawliszyn, J. A Critical Review in Calibration Methods for Solid-Phase Microextraction. Anal. Chim. Acta 2008, 627, 184.

(3) Górecki, J.; Yu, X.; Pawliszn, J. Theory of Analyte Extraction by Selected Porous Polymer SPME Fibres. Analyst 1999, 124, 643. 
(4) Du, F.; Alam, M. N.; Pawliszyn, J. Aptamer-Functionalized Solid Phase MicroextractionLiquid Chromatography/tandem Mass Spectrometry for Selective Enrichment and Determination of Thrombin. Anal. Chim. Acta 2014, 845, 45.

(5) Risticevic, S.; Pawliszyn, J. Solid-Phase Microextraction in Targeted and Nontargeted Analysis: Displacement and Desorption Effects. Anal. Chem. 2013, 85, 8987.

(6) Gionfriddo, E.; Souza-Silva, E. A.; Pawliszyn, J. Headspace versus Direct Immersion Solid Phase Microextraction in Complex Matrixes: Investigation of Analyte Behavior in Multicomponent Mixtures. Anal. Chem. 2015, 87, 8448.

(7) Koziel, J.; Jia, M.; Pawliszyn, J. Air Sampling with Porous Solid-Phase Microextraction Fibers. Anal. Chem. 2000, 72, 5178.

(8) Zhang, H.; Davison, W. Use of Diffusive Gradients in Thin-Films for Studies of Chemical Speciation and Bioavailability. Environ. Chem. 2015, 12, 85.

(9) Seethapathy, S.; Gorecki, T.; Li, X. Passive Sampling in Environmental Analysis. J Chromatogr A 2008, 1184, 234.

(10) Szulejko, J. E.; Kim, K.-H. A Review of Sampling and Pretreatment Techniques for the Collection of Airborne Amines. TrAC Trends Anal. Chem. 2014, 57, 118.

(11) Roll, I. B.; Halden, R. U. Critical Review of Factors Governing Data Quality of Integrative Samplers Employed in Environmental Water Monitoring. Water Res 2016, 94, 200.

(12) Davison, W.; Zhang, H. Progress in Understanding the Use of Diffusive Gradients in Thin Films (DGT) : Back to Basics. Environ. Chem. 2012, 9, 1.

(13) Woolcock, P. J.; Koziel, J. A.; Johnston, P. A.; Brown, R. C.; Broer, K. M. Analysis of Trace Contaminants in Hot Gas Streams Using Time-Weighted Average Solid-Phase Microextraction: Pilot-Scale Validation. Fuel 2015, 153, 552.

(14) Martos, P. A.; Pawliszn, J. Time-Weighted Average Sampling with Solid-Phase Microextraction Device: Implications for Enhanced Personal Exposure Monitoring to Airborne Pollutants. Anal. Chem. 1999, 71, 1513.

(15) Schupfer, P. Y.; Huynh, C. K. Solid Phase Microextraction as a Short-Term Sampling Technique for BTEX Occupational Exposure. J Occup Env. Hyg 2008, 5, 490.

(16) Alam, M. N.; Pawliszyn, J. Numerical Simulation and Experimental Validation of Calibrant-Loaded Extraction Phase Standardization Approach. Anal. Chem. 2016, 88, 8632.

(17) Chen, Y.; Koziel, J. A.; Pawliszn, J. Calibration for On-Site Analysis of Hydrocarbons in Aqueous and Gaseous Samples Using Solid-Phase Microextraction. Anal. Chem. 2003, 75, 6485. 
(18) Sukola, K.; Koziel, J.; Augusto, F.; Pawliszn, J. Diffusion-Based Calibration for SPME Analysis of Aqueous Samples. Anal. Chem. 2001, 73, 13.

(19) Khaled, A.; Pawliszyn, J. Time-Weighted Average Sampling of Volatile and SemiVolatile Airborne Organic Compounds by the Solid-Phase Microextraction Device. J. Chromatogr. A 2000, 892, 455.

(20) Ouyang, G.; Zhao, W.; Bragg, L.; Qin, Z.; Alaee, M.; Pawliszn, J. Time-Weighted Average Water Sampling in Lake Ontario with Solid-Phase Microextraction Passive Samplers. Environ. Sci. Technol. 2007, 41, 4026.

(21) Chen, Y.; Pawliszn, J. Time-Weighted Average Passive Sampling with a Solid-Phase Microextraction Device. Anal. Chem. 2003, 75, 2004.

(22) Jiang, R.; Xu, J.; Lin, W.; Wen, S.; Zhu, F.; Luan, T.; Ouyang, G. Investigation of the Kinetic Process of Solid Phase Microextraction in Complex Sample. Anal. Chim. Acta 2015, 900, 111.

(23) Alam, M. N.; Ricardez-Sandoval, L.; Pawliszyn, J. Numerical Modeling of Solid-Phase Microextraction: Binding Matrix Effect on Equilibrium Time. Anal. Chem. 2015, 87, 9846.

(24) Sajjadi, H.; Tavakoli, B.; Ahmadi, G.; Dhaniyala, S.; Harner, T.; Holsen, T. M. Computational Fluid Dynamics (CFD) Simulation of a Newly Designed Passive Particle Sampler. Env. Pollut 2016, 214, 410.

(25) Semenov, S. N.; Koziel, J. A.; Pawliszyn, J. Kinetics of Solid-Phase Extraction and SolidPhase Microextraction in Thin Adsorbent Layer with Saturation Sorption Isotherm. J Chromatogr A 2000, 873, 39.

(26) Rasoulian, S.; Ricardez-Sandoval, L. A. Uncertainty Analysis and Robust Optimization of Multiscale Process Systems with Application to Epitaxial Thin Film Growth. Chem. Eng. Sci. 2014, 116, 590.

(27) Ricardez-Sandoval, L. A. Current Challenges in the Design and Control of Multiscale Systems. Can. J. Chem. Eng. 2011, 89, 1324.

(28) Louch, D.; Motlagh, S.; J., P. Dynamics of Organic Compound Extraction from Water Using Liquid-Coated Fused Silica Fibers. Anal Chem 1992, 64, 1187.

(29) Murthy, C. R.; Armani, A. M. Mass Transport Effects in Suspended Waveguide Biosensors Integrated in Microfluidic Channels. Sens. Basel 2012, 12, 14327.

(30) Squires, T. M.; Messinger, R. J.; Manalis, S. R. Making It Stick: Convection, Reaction and Diffusion in Surface-Based Biosensors. Nat. Biotechnol. 2008, 26, 417.

(31) Ramus, K.; Kopinke, F. D.; Georgi, A. Sorption-Induced Effects of Humic Substances on Mass Transfer of Organic Pollutants through Aqueous Diffusion Boundary Layers: The Example of Water/air Exchange. Env. Sci Technol 2012, 46, 2196. 
(32) Gouliarmou, V.; Smith, K. E.; de Jonge, L. W.; Mayer, P. Measuring Binding and Speciation of Hydrophobic Organic Chemicals at Controlled Freely Dissolved Concentrations and without Phase Separation. Anal. Chem. 2012, 84, 1601.

(33) Zou, Y.; Zheng, W. Modeling Manure Colloid-Facilitated Transport of the Weakly Hydrophobic Antibiotic Florfenicol in Saturated Soil Columns. Environ. Sci. Technol. 2013, 47, 5185.

(34) Mocho, P.; Nicolle, J.; Desauziers, V. Modelling of Adsorption Kinetics and Calibration Curves of Gaseous Volatile Organic Compounds with Adsorptive Solid-Phase Microextraction Fibre: Toluene and Acetone for Indoor Air Applications. Anal Bioanal Chem 2008, 392, 97. 
For TOC only

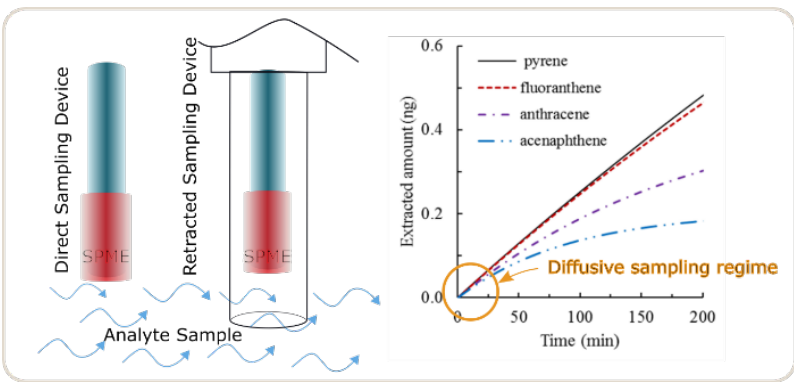

32 of 29 